\title{
Removal of Triolein Lipid From Aqueous System by Molecularly Imprinted Chitosan and Its Derivative
}

\author{
Soma Chakraborty* and Xyza Jane Templonuevo \\ Department of Chemistry, School of Science and Engineering, Loyola Schools, Ateneo de Manila University, \\ Loyola Heights, Quezon City 1108
}

\begin{abstract}
Chitosan was molecularly imprinted to remove triolein (a model lipid triacylglyceride) from water. Molecularly-imprinted chitosan (chitosan-MIP) was synthesized by crosslinking it with glutaraldehyde in the presence of triolein as the template at $50^{\circ} \mathrm{C}$ for $2 \mathrm{~h}$. MIPs of octanoyl derivative of chitosan(Oct-MIP) were also prepared by similar method. Octanoyl chitosan was synthesized by $\mathrm{N}$-acylation of chitosan using octanoyl chloride at room temperature for $12 \mathrm{~h}$. Contact angle measurements of water droplet on chitosan and octanoyl chitosan revealed increased hydrophobicity of octanoyl derivative of chitosan. FTIR spectroscopy was used to characterize the derivative and the MIPs. All the synthesized polymers. Oct-MIP and chitosanMIP could imprint approximately $77 \%$ and $66 \%$ of triolein template, respectively. Binding experiments revealed that Oct-MIP exhibit higher triolein binding capacity than corresponding non-acylated polymers. In $6 \mathrm{~h}, 1 \mathrm{mg}$ of Oct-MIP and chitosan-MIP could rebind $534.50 \mu \mathrm{g}$ and $380.35 \mu \mathrm{g}$ of triolein respectively. Non Imprinted octanoyl chitosan and chitosan bound $272 \mu \mathrm{g}$ and $198.24 \mu \mathrm{g}$ triolein respectively. Both types of MIPs could also bind a triolein analog (1,3-dioleoyl-2-palmitoyl glycerol).
\end{abstract}

Keywords: chitosan; $N$-acylated chitosan; molecularly imprinted polymer; triolein; lipid pollution

\section{INTRODUCTION}

Lipids are organic pollutants mainly from slaughterhouses and meat processing facilities are present at significant concentrations in wastewater. When they are present in significant quantity, they diminish the amount of dissolved oxygen in water as they need oxygen during degradation (Salam et al., 2012; Campo et al., 2012). Further, lipid films and emulsified lipids may interact with debris and settle to the bottom of the water body (Crump-Wiesner et al., 1975). Depleted amount of dissolved oxygen has been shown to cause disruption of the aquatic ecosystem (Bucas et al., 2002; Christie et al., 1977; Russell et al., 1978). Use of hydrolytic enzymes (Cammarota et al., 2006; El-Gawad 2014), aerobic and anaerobic biodegradation (Chipasa et al. 2006; Jensen et al., 2015) have been identified as viable remediations for removal of lipid pollutants from water bodies. However, long-chain lipids have been found to inhibit the activity of the microorganisms responsible for anaerobic remedy (Lalman et al., 2000; Lalman et al., 2001). This compromises the efficiency of anaerobic biodegradation for the treatment of lipid-rich wastewater. Hence longchain fatty acid components must be sequestered prior to anaerobic remediation. As of now there is hardly any report of using an efficient vehicle for selective sequestration of long chain lipids.

Molecularly imprinted polymers (MIPs) can be explored for the selective removal of large lipids 
by imprinting them with the desired lipid as the template. They have been utilized for selective removal of organic water pollutants such as diclofenac (Dai et al., 2011), estrogenic compounds (Lucci et al., 2011), polycyclic aromatic hydrocarbons (Krupadam et al., 2010) and polychlorinated biphenyl analogs (Sikiti et al., 2014).

One of the potential raw materials for MIP synthesis is chitosan due to its low cost and high natural abundance. Some studies have already established that chitosan MIP can efficiently remove organic pollutants from water (Zhang et al., 2017; Wang et al.,2018). Chitosan MIPs have been also explored as absorbent of organic pollutants from various sources. including dibenzenethiophene (Aburto et al., 2004; Chang et al.,2008), dyes(Lazaridis et al., 2007) and perflurooctane sulfonate (Yu et al., 2008). Though there are several studies on use of chitosan MIPs as absorbent for small molecules, the ability of chitosan MIPs and its derivatives to bind to large moieties such as triolein is yet to be explored. This study investigated the ability of MIPs of chitosan and octyl derivative of chitosan(OctMIP) to bind to triolein from water to explore the potential of chitosan to remove lipid pollutants from water bodies.

\section{EXPERIMENTAL}

Chemicals. Chitosan (medium molecular weight - 190-310 kDa, 200.000 cps, DDA 75-85\%), octanoyl chloride $(99 \%)$, triolein $(\geq 99 \%)$, Triton X-114 and 1,3-dioleoyl-2-palmitoylglycerol ( $\geq 99 \%)$ were purchased from Sigma Aldrich, glutaraldehyde ( $50 \%$ aqueous solution) was obtained from Merck, The solvents absolute ethanol, glacial acetic acid, isopropanol and n-hexane (AR, RCL Labscan) were purchased from Labscan and used as received.

Instruments. UV-visible (UV-vis) Spectroscopy. UVvis analyses to quantify imprinting and binding of trioelin to MIPs and NIPs were conducted using Shimadzu UV-1800 spectrophotometer and processed using UV Probe 2.35.

FTIR Spectroscopy. IR analyses of chitosan derivative and crosslinked chitosan were performed using Shimadzu FTIR - IR Affinity 1 and IR Affinity 2 at a resolution of $4.0 \mathrm{~cm}^{-1}$ and with 40 scans conducted per sample. Data were processed using Shimadzu IR Solution 1.30 .

Contact Angle Measurement. The change in hydrophobicity of chitosan upon acylation was observed by contact angle measurement of deionized water on chitosan and octanoyl chitosan films.
The films were produced by dissolving $0.01 \mathrm{~g}$ of each polymer in $0.5 \mathrm{~mL}$ of $1 \%$ acetic acid and then spincoating $60 \mu \mathrm{L}$ of the solution onto $1 \mathrm{~cm} \times 1 \mathrm{~cm}$ glass slides (Laurell WS-650Mz-23NPP spin coater). The films were washed with $0.2 \mathrm{M} \mathrm{NaOH}$ and then with distilled water until they were neutral and dry. The image of water droplet on the film was captured using a digital video camera (Sony NP F300) and processed using the software ImageJ Contact angles for 3 locations on each film were measured and 3 films per polymer type was analyzed

Methodology. Synthesis of Octanoyl Chitosan. The acylation procedure used was adapted from the work of Remant Bahadur et al. (Remant Bahadur et al., 2006) with some modifications. Chitosan $(0.5 \mathrm{~g}$, $3.1 \mathrm{mmol}$ ) was dissolved in $50 \mathrm{~mL}, 1 \%$ aqueous acetic acid solution. Octanoyl chloride $(319 \mu \mathrm{L}$, $1.86 \mathrm{mmol}$ ) was added under constant agitation. The mixture was then diluted to $500 \mathrm{~mL}$ with distilled water and subsequently neutralized with $0.2 \mathrm{M}$ $\mathrm{NaOH}$ every 10 min for 2 hours or until the $\mathrm{pH}$ was constant at 7.00. The mixture was allowed to stir for $12 \mathrm{~h}$ at room temperature. The white suspension was centrifuged and collected. This acylated product was repeatedly washed with $20 \mathrm{~mL}$ absolute ethanol, and centrifuged to collect the final product. The product was then dried to constant weight under vacuum and characterized by FTIR and elemental analysis. The ethanol washes were subjected to FTIR analysis for detection of any unreacted octanoyl chloride and for determination of the number of washes required to completely remove the excess acylating agent.

Synthesis of Chitosan-MIP and Octanoyl ChitosanMIP (Oct-MIP). Synthesis of chitosan-MIP was adapted from the procedure by Aburto \& Le Borgne (Aburto et al.,2004) with some modifications. Chitosan (0.1g, $0.62 \mathrm{mmol})$, was dissolved in $8 \mathrm{~mL} 1 \%$ acetic acid aqueous solution. To it, $40 \mu \mathrm{L}$ surfactant (Triton X-114) and $0.15 \mathrm{~mL}$ of triolein were added. The system was stirred for $30 \mathrm{~min}$ and then $2 \mathrm{~mL}$ of $0.031 \mathrm{M}$ aqueous glutaraldehyde solution was added. The reaction was allowed to proceed for $2 \mathrm{~h}$ at $50^{\circ} \mathrm{C}$.

At the end of the reaction period, $20 \mathrm{~mL}$ of $70: 30$ $(\mathrm{v} / \mathrm{v})$ hexane-isopropanol was added to the viscous system and stirred for $5 \mathrm{~min}$. This hexane-isopropanol mixture was then recovered by centrifugation and subjected to UV-vis analysis for the quantification of unbound triolein and for the detection of the presence of unreacted glutaraldehyde by IR analysis.

The non-imprinted polymer (chitosan-NIP) was prepared using the same procedure but without the addition of triolein. The synthesis of octanoyl chitosan-MIP (oct-MIP) and its non-imprinted 
counterpart (oct-NIP) also followed the same process but octanoyl chitosan was used instead of chitosan.

Triolein Removal. To generate the empty cavities inside the MIPs, the bound triolein was extracted by equilibrating the MIPs in $20 \mathrm{~mL} 70: 30(\mathrm{v} / \mathrm{v})$ hexaneisopropanol and then in hexane for $30 \mathrm{~min}$ and then recovering the MIPs by centrifugation. The process was repeated several times till no triolein was detected in the washing using UV-visible spectroscopy.

Triolein Binding. Triolein rebinding was studied as a function of time. Chitosan-MIP or oct-MIP (3mg) was added to a dispersion of $2.7 \mu \mathrm{L}$ triolein, in $1 \mathrm{~mL}$ water, and subjected to constant stirring for certain time intervals. Each sample was centrifuged and the aqueous supernatant was decanted. The polymer was then given a quick wash of hexane to remove unbound or loosely bound template to the surface. The triolein in both fractions were quantified by UV-vis spectroscopy to find the amount of triolein that remained unbound to the MIP after a particular contact time.

Binding with a Triolein Analog. MIPs were allowed to bind 1,3-dioleoyl-2-palmitoylglycerol(OPO) by equilibrating the MIPs with OPO solution for $6 \mathrm{~h}$ following the procedure that was adopted for the rebinding study of triolein.

\section{RESULTS AND DISCUSSION}

Synthesis and Characterization of Octanoyl Chitosan. Chitosan was reacted with octanoyl chloride to increase its interaction with the hydrophobic template, triolein. The reaction scheme is shown in Figure 1.

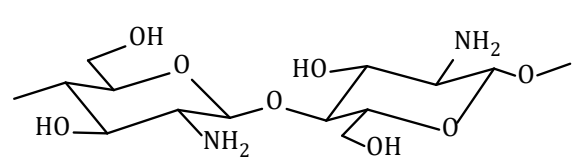

chitosan

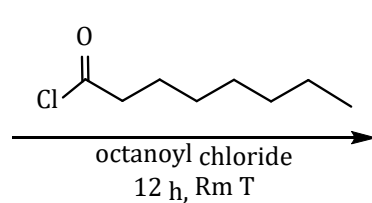

$12 \mathrm{~h}, \mathrm{Rm} \mathrm{T}$

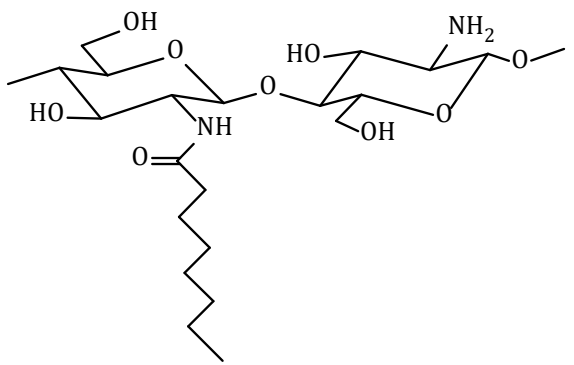

octanoyl chitosan

Figure 1. N-Acylation of Chitosan Using Octanoyl Chloride.

The product of the acylation reaction for $12 \mathrm{~h}$ was washed with absolute ethanol. FTIR analysis shows that four ethanol washes were required to remove unreacted octanoyl chloride. The IR spectrum of the 4th wash resembled that of the solvent alone.

Acylation of chitosan was confirmed using IR spectroscopy. The IR spectrum of octanoyl chitosan (Figure 2) showed increased intensity of: the alkyl stretch at 2880-2980 $\mathrm{cm}^{-1}$ due to the added $\mathrm{CH}_{2}$ and $\mathrm{CH}_{3}$ groups as compared to chitosan alone.

Contact angle measurement was performed to determine the effect of acylation on the hydrophobicity of chitosan. A drop of deionized water was placed on native chitosan and octanoyl chitosan films to measure the contact angle. As given in Table 1, higher contact angle for octanoyl chitosan indicates its enhanced hydrophobic character relative to chitosan.

Synthesis and Characterization of ChitosanMIP and Oct-MIP. Chitosan was molecularly imprinted by crosslinking it with glutaraldehyde in the presence of the triolein. Crosslinking was confirmed by the presence of an imine $(\mathrm{C}=\mathrm{N})$ peak

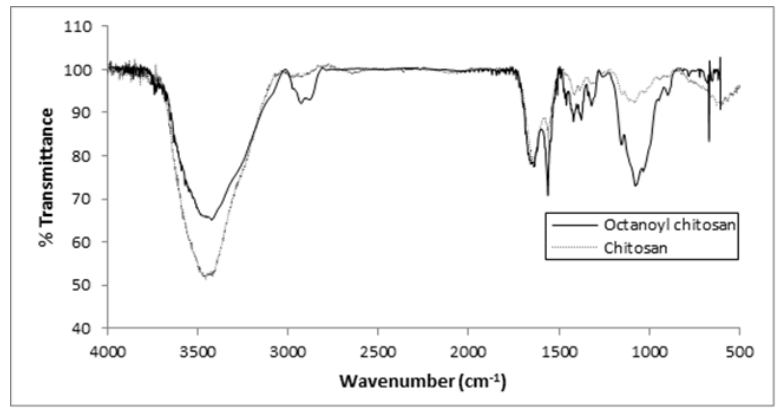

Figure 2. FTIR Spectra of Octanoyl Chitosan and Chitosan.

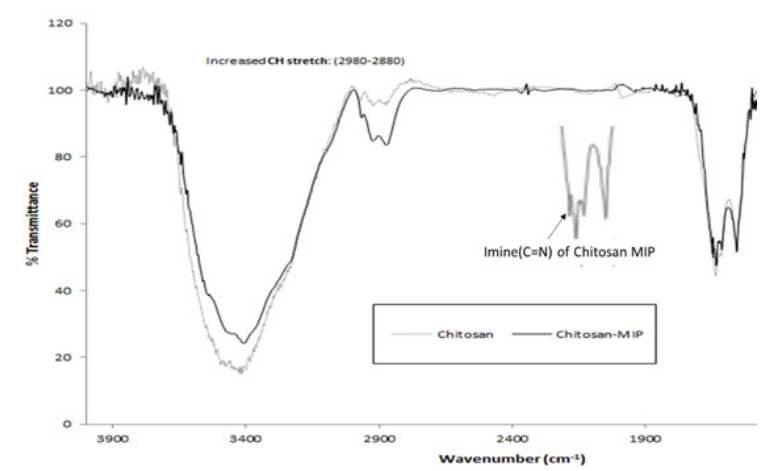

Figure 3. FTIR Spectra of Chitosan and Chitosan MIP. 
Table 1. Contact Angle of Water Droplet on Chitosan and Octanoyl Chitosan Film

\begin{tabular}{ccc}
\hline Sample & Contact Angle $\left(^{\circ}\right)$ & $\begin{array}{l}\text { Image of } \mathrm{H}_{2} \mathbf{O} \\
\text { Drop on Film }\end{array}$ \\
\hline Chitosan & 57.5 & \\
\hline $\begin{array}{c}\text { Octanoyl } \\
\text { chitosan }\end{array}$ & 76.4 & \\
\hline
\end{tabular}

at $1655 \mathrm{~cm}^{-1}$ in the IR spectrum of chitosan-MIP as shown in Figure 3. The FTIR spectrum of 70:30 hexane-isopropanol wash also did not show the presence of glutaraldehyde indicating completion of the crosslinking at the given reaction conditions. The imine $(\mathrm{C}=\mathrm{N})$ peak was also visible in the FTIR spectrum of Oct-MIP which has not been included as the part of the figure.

Extraction of Bound Triolein from Chitosan-MIP and Oct-MIP. To remove triolein from the MIPs, they were washed repeatedly with 70:30 hexaneisopropanol, and then with pure hexane alone. Pure hexane was not used throughout since decreasing the polarity of the solvent was found to be less efficient in removing the surfactant. The washings were subjected to UV-vis spectroscopy analysis. The peak at $277 \mathrm{~nm}$ which corresponds to the surfactant and triolein peak was monitored for each washing. As shown in Figure 4, 16 washings were required for their removal.

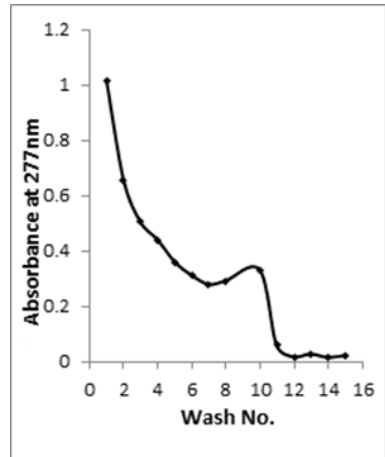

Figure 4. UV-vis Spectroscopy of Washings Used to Remove the Surfactant and Template from the MIP.

Triolein Imprinting Efficiency. As quantified from the UV-vis spectra, oct- MIP could bind $105.51 \pm 11.60 \mathrm{mg}(77.29 \%)$ of triolein whereas chitosan-MIP could bind $92.40 \pm 5.59 \mathrm{mg}(66.41 \%)$. Higher binding efficiency of oct-MIP is attributed to hydrophobic interaction between octanoyl chitosan and the hydrophobic template.
Triolein Binding. Binding of triolein to chitosanMIP, Oct-MIP and the corresponding NIPs was performed as function of time. The binding capacity (Q) of each polymer is defined by the following equation:

$$
Q=\frac{\text { mass of triolein bound to the MIP/NIP }(\mu \mathrm{g})}{\text { mass of polymer }(\mathrm{mg})}
$$

The binding profiles are shown in Figure 5. Results show higher Q values for MIPs as compared to the NIPs. This is attributed to imprinting of the template that occured upon crosslinking of the polymer in the presence of triolein, creating cavities in the polymer that exhibit conformational memory for the template. Furthermore, octanoyl modification of chitosan contributed towards enhanced triolein binding. After $6 \mathrm{~h}$ of equilibration, triolein binding of oct-NIP and oct-MIP were $26 \%$ and $41 \%$ higher than their corresponding non-acylated chitosan. Thus, affinity of a substrate for a target molecule can be increased by the formation of MIP as well as the chemical modification of the substrate.

Figure 5 also reveals that for chitosan-MIP, chitosanNIP and Oct-NIP there was no significant increase in binding after $2 \mathrm{~h}$ of equilibration, probably due to the saturation of hydrogels with the triolein molecules within $2 \mathrm{~h}$. However oct-MIP showed marked increase in binding till the equilibration time reached $6 \mathrm{~h}$, proving that the hydrogel got saturated at higher concentration of triolein due to its stronger affinity for the target molecule.

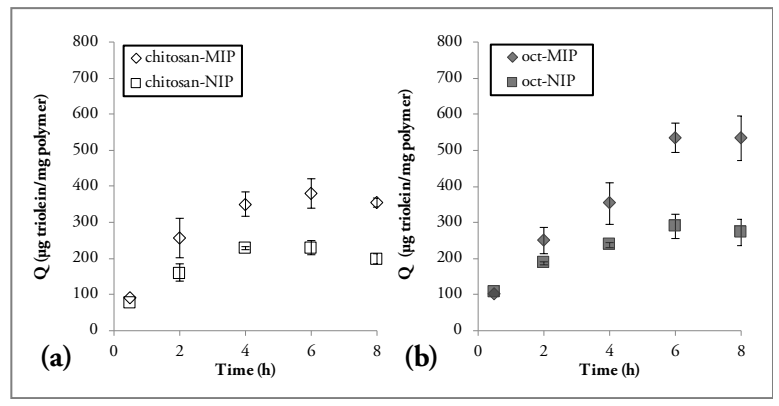

Figure 5. Triolein Bound by Chitosan MIP, Oct MIP and Their NIP Counterparts as a Function of Time.

The study by Ahmad et al. (Ahmad et al. 2005) stated that $0.5 \mathrm{~g}$ of chitosan flakes and powder could remove $1.30 \mathrm{~g}$ and $1.98 \mathrm{~g}$ of lipid respectively from water, whereas this study reveals that only $0.003 \mathrm{~g}$ of chitosan-MIP and oct-MIP could remove $1.14 \mathrm{~g} / \mathrm{L}$ and $1.60 \mathrm{~g} / \mathrm{L}$ of lipid respectively under similar initial lipid concentrations as reported by Ahmad et al. Thus, use of chitosan and its derivatives in the form of MIP can be considered as more efficient absorbent of lipid wastes from water bodies. 
$Q$ values were used to calculate the imprinting factor. Imprinting factor $(\alpha)$ is defined as following.

$$
\alpha=\frac{\mathrm{Q}_{\mathrm{MIP}}}{\mathrm{Q}_{\mathrm{NIP}}}
$$

The imprinting factor for both types of MIP systems has been found to increase with time (Figure 6). In the case of MIPs, apart from surface adsorption, more triolein could gain access to the binding sites within the imprinted polymer over time whereas for NIPs, surface adsorption was the only binding factor. This observation further reinstates that formation of MIP aids in enhanced binding of triolein.

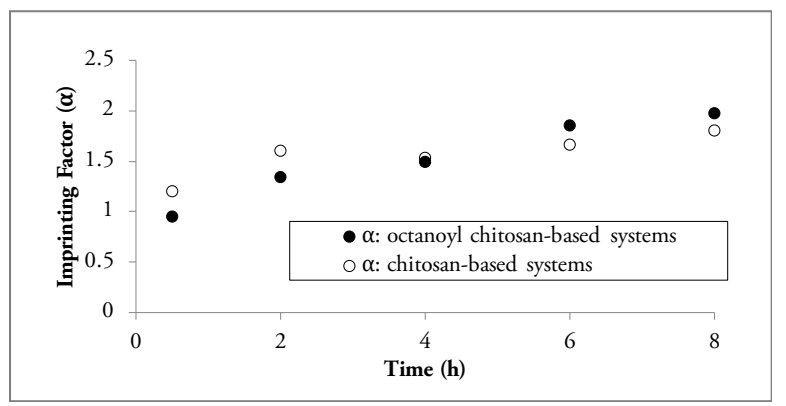

Figure 6. Imprinting Factor as a Function of Time.

Binding with a Triolein Analog. Triolein and 1,3-dioleoyl-2-palmitoylglycerol(OPO) differ only at the fatty acid esterified to the hydroxyl group at carbon-2 of glycerol as shown in Figure 7.

Figure 8 shows that both chitosan-MIP and oct-MIP could also bind OPO though the binding capacity towards OPO is marginally lower. This result reflects that these MIPs can not only be used for the removal of triolein but also other triacylglycerides from wastewater samples, though the binding capacity will be maximum for template specific triolein.

\section{CONCLUSIONS}

The study established that both chitosan-MIP and Oct-MIP can imprint triolein and can efficiently bind to it when the MIPs and triolein are dispersed in water. Chitosan-MIP and Oct-MIP had higher affinity for triolein than the non imprinted counterparts. Binding capacity of Oct-MIP was higher than chitosan-MIP due to the enhanced hydrophobic interaction between the MIP and the substrate. Apart from triolein the MIPs could bind to its analog 1,3-dioleoyl-2-palmitoylglycerol.

Since chitosan is one of the abundant natural polymers and has the potential to bind to lipids such as triolein and 1,3-dioleoyl-2-palmitoylglycerol in water, of which MIP is the most efficient form, chitosan and its octanoyl derivative in the form

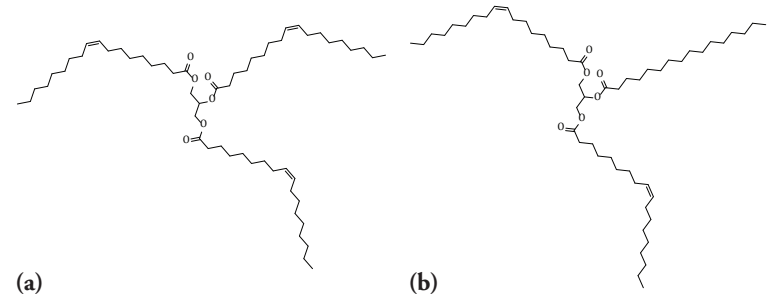

Figure 7. Structure of (a) Triolein (b) 1,3-Dioleoyl-2palmitoylglycerol.

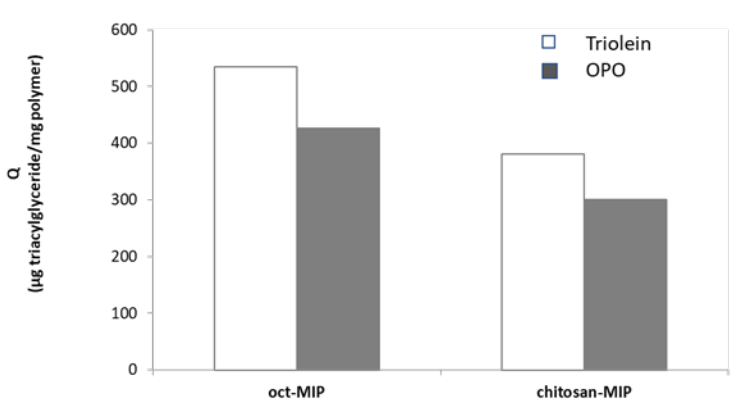

Figure 8. Binding of Triacylglycerides to Chitosan-MIP and Oct-MIP.

of MIP can be explored further as a low-cost and efficient system to remove lipid waste from water bodies.

\section{ACKNOWLEDGEMENT}

The authors would like to express our gratitude to the Accelerated Science and Technology Human Resource Development Program (ASTHRDP) of the Department of Science and Technology for the scholarship and research grant, Reynaldo Coria of the De La Salle University for SEM analyses.

\section{REFERENCES}

Aburto J, Le Borgne S. Selective adsorption of dibenzothiophene sulfone by an imprinted and stimuli-responsive chitosan hydrogel. Macromolecules. 2004; 37(8): 2938-2943.

Ahmad AL, Sumathi S, Hameed BH. Adsorption of residue oil from palm oil mill effluent using powder and flake chitosan: Equilibrium and kinetic studies. Water Res. 2005; 39(12): 2483-2494.

Bucas G, Saliot A. Sea transport of animal and vegetable oils and its environmental consequences. Mar. Pollut. Bull. 2002; 44(12): 1388-1396.

Cammarota MC, Freire DMG. A review on hydrolytic enzymes in the treatment of wastewater with high oil and grease content. Bioresour Technol. 2006; 97(17): 2195-2210.

Campo P, Zhao Y, Suidan MT, Venosa AD. Aerobic 
fate and impact of canola oil in aquatic media. Clean Technol. Environ. Policy. 2012; 14(1): 125-132.

Chang Y, Zhang L, Ying H, Li Z, Lv H, Ouyang P. Desulfurization of Gasoline using Molecularly Imprinted Chitosan as Selective Adsorbents. Appl. Biochem. Biotechnol. 2010; 160(2): 593-603.

Chipasa KB, Medrzycka K. Behavior of lipids in biological wastewater treatment processes. J. Ind. Microbiol. Biotechnol.2006; 33(8): 635-45.

Christie N, Moldan A. Effects of fish factory effluent on the benthic macrofauna of Saldanha Bay. Mar. Pollut. Bull. 1977; 8(2): 41-45.

Crump-Wiesner HJ, Jennings AL. Properties and effects of nonpetroleum oils. In: Proceedings of the 1975 Conference on Prevention and Control of Oil Pollution, 1975; 29-32.

Dai CM, Geissen SU, Zhang YL, Zhou XF. Selective removal of diclofenac from contaminated water using molecularly imprinted polymer microspheres. Environmental Pollution. 2011; 159(6): 16601666.

El-Gawad. Oil and grease removal from industrial wastewater using new utility approach. Advances in Environmental Chemistry. 2014. Article ID 916878, 6 pages.

Jensen PD, Yap SD, Boyle-Gotla A, Janoschka J, Carney C, Pidou M, Batstone DJ. Anaerobic membrane bioreactors enable high rate treatment of slaughterhouse wastewater, Biochemical Engineering Journal.2015; 97: 132-141.

Krupadam RJ, Khan MS, Wate SR. Removal of probable human carcinogenic polycyclic aromatic hydrocarbons from contaminated water using molecularly imprinted polymer. Water Res. 2010; 44(3): 681-688.

Lalman J, Bagley D. Anaerobic degradation and inhibitory effects of linoleic acid. Water Res. 2000; 34(17): 4220-4228.

Lalman J, Bagley D. Anaerobic degradation and methanogenic inhibitory effects of oleic and stearic acids. Water Res. 2001.; 35(12): 2975-2983.

Lazaridis NK, Kyzas GZ, Vassliou AA, Bikiaris DN. Chitosan Derivatives as Biosorbents for Basic Dyes, Langmuir 2007; 23, 7634-7643

Lucci P, Núñez O, Galceran MT. Solid-phase extraction using molecularly imprinted polymer for selective extraction of natural and synthetic estrogens from aqueous samples. J. Chromatogr. A. 2011; 1218(30): 4828-4833.

Remant Bahadur RKC, Aryal S, Bhattarai SR, Bhattarai N, Kim CH, Kim HY. Stabilization of gold nanoparticles by hydrophobically-modified polycations. J. Biomater. Sci., Polym. Ed. 2006; 17(5): 579-589.

Russell D, Carlson B. Edible oil pollution on Fanning Island. Pac. Sci. 1978; 1-15.

Salam DA, Naik N, Suidan MT, Venosa AD. Assessment of aquatic toxicity and oxygen depletion during aerobic biodegradation of vegetable oil: Effect of oil loading and mixing regime. Environ. Sci. Technol. 2012; 46(4): 2352-2359.

Sikiti P, Msagati TAM, Mamba BB, Mishra AK. Synthesis and characterization of molecularly imprinted polymers for the remediation of PCBs and dioxins in aqueous environments. J. Environ. Health Sci. Eng. 2014; 12: 82.

Wang J, Zhuang S. Removal of various pollutants from water and wastewater by modified chitosan adsorbents. Crit Rev Env Sci Tec. 2018; 47,23: 2331-2386.

Yu Q, Deng S, Yu G. Selective removal of perfluorooctane sulfonate from aqueous solution using chitosan-based molecularly imprinted polymer adsorbents. Water Research. 2008; 12: 3089-3097.

Zhang W, ZhuZ, Zhang H, Qiu Y.SelectiveRemoval of the Genotoxic Compound 2-Aminopyridine in Water Using Molecularly Imprinted Polymers Based on Magnetic Chitosan and $\beta$-Cyclodextrin. Int J Environ Res Public Health. 2017; 14(9): 991-3101. 